\title{
Detection of circulating tumor cells in pulmonary venous blood for resectable non-small cell lung cancer
}

\author{
CHAO LV $^{1 *}$, BINGTIAN ZHAO ${ }^{1 *}$, LIMIN WANG $^{2}$, PANPAN ZHANG $^{1}$, YUANYUAN MA $^{1}$, \\ YUZHAO WANG $^{1}$, NAN WU ${ }^{1}$, YING WU ${ }^{3}$ and YUE YANG $^{1}$ \\ ${ }^{1}$ Second Department of Thoracic Surgery, ${ }^{2}$ Laboratory of Clinical Research, and ${ }^{3}$ Department of Pathology, \\ Key Laboratory of Carcinogenesis and Translational Research (Ministry of Education), \\ Peking University Cancer Hospital and Institute, Beijing 100142, P.R. China
}

Received November 21, 2015; Accepted April 25, 2017

DOI: $10.3892 / \mathrm{ol} .2017 .7405$

\begin{abstract}
Intraoperative manipulation causes circulating tumor cell (CTC) shedding into the blood and accelerates metastasis in non-small cell lung cancer (NSCLC). The present study was conducted to assess the degree of dissemination resulting from surgery and to explore the biological features of CTCs. In patients with NSCLC who underwent complete resection, the pulmonary vein (PV) was isolated and stapled following thoracotomy. The number of CTCs retained per $7.5 \mathrm{ml}$ PV blood (CTC-PV) and peripheral blood were detected. Following hematopoietic cell depletion, a xenograft assay was performed using the CTC-PV. A total of 32 consecutive patients were enrolled in the study, the majority of whom had CTCs in their PV blood $(n=29,90.6 \%)$. Circulating tumor microemboli (CTM) were identified in 12 patients (37.5\%). The CTC-PV and CTM-PV counts were positively correlated with tumor size $(\mathrm{P}=0.012$ and $\mathrm{P}=0.028$, respectively). Patients with small tumors $(<3.0 \mathrm{~cm})$ also had considerable CTC-PV and CTM-PV. A total of 8 patients received platinum-based chemotherapy prior to surgery. The CTC-PV and CTM-PV counts in patients with partial response were significantly lower than those in patients with stable disease or who did not receive induction therapy $(\mathrm{P}=0.025$ and $\mathrm{P}=0.044$, respectively). The enriched CTC-PV from 3 patients were injected into 3 immunodeficient mice, and 1 mouse developed a xenograft tumor. To conclude, the present study indicates that intraoperative manipulation contributes
\end{abstract}

Correspondence to: Dr Yue Yang, Second Department of Thoracic Surgery, Key Laboratory of Carcinogenesis and Translational Research (Ministry of Education), Peking University Cancer Hospital and Institute, 52 Fucheng Road, Haidian, Beijing 100142, P.R. China

E-mail: zlyangyue@bjmu.edu.cn

*Contributed equally

Key words: non-small cell lung cancer, hematogenous spread, circulating tumor cell, circulating tumor microemboli, lobectomy, pulmonary vein ligation to the hematogenous dissemination of tumorigenic CTCs and CTM. Lobectomy is recommended for lung cancer of any tumor size and stage according to oncological principles, in addition to ligating the PV, if possible, prior to any other treatment.

\section{Introduction}

Lung cancer is the leading cause of cancer-associated mortality globally (1). Although surgical resection is considered the best chance for curing patients with early-stage disease and certain patient subsets with locally advanced disease, the prognosis for these patients is unfavorable, with poor five-year survival rates and high recurrence rates $(2,3)$. Hematogenous dissemination of circulating tumor cells (CTCs) is important in initiating metastasis and disease recurrence (4). In addition to tumor biological features, intraoperative manipulation may also cause CTC shedding into the blood and accelerate metastasis subsequent to surgery $(5,6)$.

Over the past decade, the CELLSEARCH system (Veridex LLC, Raritan, NJ, USA), based on immunomagnetic enrichment of epithelial cells and identification by fluorescently labeled antibodies (7), has been widely used to evaluate CTC numbers, and by extension, to assess treatment response and predict prognosis in cancer, including non-small cell lung cancer (NSCLC) (8-10). Using CELLSEARCH, two studies on lung cancer surgery evaluated CTC counts in pulmonary venous (PV) blood and peripheral blood during surgery $(11,12)$. Although these studies reported the presence of CTCs in PV blood (CTC-PV) and increased CTC-PV following intraoperative manipulation, whether their conclusions can be used to standardize and guide intraoperative surgical behavior for lung cancer is unclear. In addition, whether CTCs detected in PV blood are viable and tumorigenic is also unknown.

The present study was conducted using CELLSEARCH to assess the extent to which surgical manipulation causes tumor cell dissemination, to analyze the associated risk factors and to explore the biological features of tumor cells shed in the blood. The present study aimed to provide evidence for confirming oncological principles in lung cancer surgery and guiding perioperative treatment. 


\section{Materials and methods}

Patients. The present study corresponds to a prospective study conducted at the Second Department of Thoracic Surgery of Peking University Cancer Hospital (Beijing, China). Between October 2013 and March 2014, a total of 33 consecutive patients were enrolled in the present study, including 32 patients with lung cancer and 1 with pulmonary hamartoma. In total, 18 malignant tumors were diagnosed by bronchoscopy or CT-guided needle biopsy prior to surgery and the corresponding patients underwent lobectomy; the remaining patients, including the patient with hamartoma, did not undergo wedge resection for biopsy prior to lobectomy during surgery since the tumor was either too large or too close to the hilum. Resection consisted of 5 pneumonectomies (15.2\%), 3 sleeve lobectomies (9.1\%), 1 bilobectomy (3.0\%) and 24 lobectomies (72.7\%). All cases met complete resection (R0) criteria (13). The 32 patients with lung cancer consisted of 19 males (59\%) and 13 females (41\%), with the age ranging between 32 and 78 years (mean, $59.5 \pm 8.7$ years). The patient clinical characteristics are listed in Table I. Prior to surgery, brain magnetic resonance imaging studies, abdomen and supraclavicular lymph node ultrasound examination, and bone scans were performed to exclude distant metastases. Positron emission tomography (PET)-computed tomography (CT) was used as a diagnostic and staging method. Patients were excluded if they had concurrent or prior malignancy treated within the previous 5 years. The Institutional Ethics Board of Peking University Cancer Hospital approved the study protocol, and patients who participated in the study provided written informed consent.

Clinical information. The same surgical team performed all surgeries, and the following clinical information was carefully recorded in detail: Age, sex, histological type, tumor site, visceral pleural involvement, vessel invasion, tumor size and primary tumor standard uptake value (SUV). The pathological stage was determined according to the 7th edition of the tumor-node-metastasis (TNM) classification system (3). Perioperative treatment, which included neoadjuvant chemotherapy, adjuvant chemotherapy and targeted therapy, was also recorded. Clinical response to neoadjuvant chemotherapy was evaluated by $\mathrm{CT}$ according to the Response Evaluation Criteria in Solid Tumors (14). Histopathological response to neoadjuvant chemotherapy was assessed according to the percentage of residual tumor, which was estimated using hematoxylin and eosin (H\&E)-stained slides of gross residual tumor sections. The result for each slide was averaged to obtain a mean value. Follow-up information was collected every 3 months within 2 years after surgery.

Blood collection. All patients underwent lobectomy, sleeve lobectomy, bilobectomy or pneumonectomy directly. Neither segmentectomy nor wedge resection was performed during the operation (Fig. 1A). For accurate N staging (15), systematic mediastinal lymph node dissection, and lobar, segmental and subsegmental node resection were performed. Following thoracotomy, the PV was first isolated and stapled using Endo GIA (Covidien Surgical Solutions, Boulder, CO, USA). When the lung was resected, PV blood samples were immediately obtained with a needle (syringe) inserted into the vein (Fig. 1B). Next, the blood sample was gently injected into a CellSave Preservative Tube (Janssen Diagnostics, LLC, Raritan, NJ, USA). Peripheral blood samples were also collected from the radial artery opposite to the surgical side during the operation, which were referred to as CTC-peri and served as controls.

Determination of CTCs and circulating tumor microemboli (CTM). Blood samples $(7.5 \mathrm{ml})$ were collected in 10-ml CellSave Preservative Tubes (Janssen Diagnostics, LLC), stored at room temperature and processed within $96 \mathrm{~h}$ of collection. CTCs were evaluated quantitatively using the USA Food and Drug Administration (FDA)-approved CELLSEARCH (Janssen Diagnostics, LLC) as described previously (7) and without knowledge of the patients' clinical characteristics. CTCs were defined as epithelial cell adhesion molecule-isolated cells with round to oval morphology and DAPI-stained nuclei expressing cytokeratins (CKs) 8, 18 and 19 but not the white blood cell-surface marker cluster of differentiation 45 . To avoid identifying mitotic CTC as microemboli, CTM were identified as CTC clusters containing $\geq 3$ distinct nuclei (16). Fig. 1C depicts the representative images of CTCs and CTM from two patients.

CTC isolation and tumorigenic assay. To explore the tumorigenic features of CTC-PV, blood samples were collected from several patients if they had residual PV blood after CELLSEARCH analysis. A total of three of 4-6-week-old female non-obese diabetic/severe combined immunodeficient (Nod-SCID) mice weighing $\sim 20 \mathrm{~g}$ were purchased from Beijing Vital River Laboratory Animal Technology Co., Ltd. (Beijing, China) and maintained in specific pathogen-free conditions $\left(19-22^{\circ} \mathrm{C}, 12 \mathrm{~h}\right.$ light and sterile food and water). Hematopoietic cells were depleted according to the RosetteSep ${ }^{\mathrm{TM}}$ Human Circulating Epithelial Tumor Cell Enrichment Cocktail procedure (cat. no. 15167; Stemcell Technologies, Inc., Vancouver, BC, Canada). Next, cells were mixed with Matrigel and directly injected subcutaneously into the flanks of these immunodeficient mice. The Peking University Cancer Hospital and Institute Animal Care and Use Committee approved the animal protocols.

Xenograft tumor analysis. Xenograft tumors were collected, fixed in formalin and paraffin-embedded, and 4- $\mu \mathrm{m}$ thick sections were obtained immediately prior to $H \& E$ staining. To identify the histological types and to analyze the expression of drug resistance-associated proteins in the xenograft model, the Department of Pathology of Peking University Cancer Hospital performed an immunohistochemistry (IHC) staining series and compared the primary tumor of the patients with the xenograft tumor. Antibodies against the following proteins were used: CK7 (cat. no. IR61961; dilution, 1:50), CK20 (cat. no. IR77761; dilution, 1:50), excision repair cross-complementing 1 (cat. no. IR09161; dilution, 1:300), thymidylate synthase (cat. no. M361401; dilution, 1:300), topoisomerase II (cat. no. M718601; dilution, 1:100), epidermal growth factor receptor (cat. no. M729829; dilution, 1:200; all from Dako; Agilent Technologies, Inc., Santa Clara, CA, USA), ribonucleotide reductase M1 (cat. no. ZA-0373; dilution, 1:100), thyroid nuclear factor-1 (cat no. ZM-0250; dilution, 1:50), 
Table I. Patient characteristics and correlation with CTCs/CTM.

\begin{tabular}{|c|c|c|c|c|}
\hline \multirow[b]{2}{*}{ Characteristics } & \multirow{2}{*}{$\begin{array}{l}\text { No. of patients with } \\
\text { lung cancer }(n=32)\end{array}$} & \multicolumn{3}{|c|}{ P-value } \\
\hline & & CTC-peri & CTCs-PV & CTMs-PV \\
\hline \multicolumn{5}{|l|}{ Sex } \\
\hline Male & $19(59 \%)$ & 0.668 & 0.050 & 0.440 \\
\hline Female & $13(41 \%)$ & & & \\
\hline Age (range) & 32-78 (years) & 0.219 & 0.664 & 0.829 \\
\hline \multicolumn{5}{|l|}{ Histological type } \\
\hline Adenocarcinoma & $16(50 \%)$ & 0.442 & 0.734 & 0.811 \\
\hline Non adenocarcinoma & $16(50 \%)$ & & & \\
\hline \multicolumn{5}{|l|}{ Tumor site } \\
\hline Central & $8(25 \%)$ & 0.764 & 0.281 & 0.789 \\
\hline Peripheral & $24(75 \%)$ & & & \\
\hline \multicolumn{5}{|c|}{ Visceral pleural involvement } \\
\hline Yes & $6(19 \%)$ & 0.182 & 0.176 & 0.331 \\
\hline No & $26(81 \%)$ & & & \\
\hline \multicolumn{5}{|l|}{ Vessel invasion } \\
\hline Yes & $6(19 \%)$ & 0.182 & 0.059 & 0.232 \\
\hline No & $26(81 \%)$ & & & \\
\hline Tumor size range $(\mathrm{cm})$ & $1.5-9.0$ & 0.016 & 0.012 & 0.028 \\
\hline \multicolumn{5}{|l|}{ Lymph node metastasis } \\
\hline Yes & $13(41 \%)$ & 0.143 & 0.038 & 1.000 \\
\hline No & $19(59 \%)$ & & & \\
\hline \multicolumn{5}{|c|}{ Pathological TNM staging } \\
\hline I & $13(41 \%)$ & 0.149 & 0.048 & 0.641 \\
\hline II & $12(37 \%)$ & & & \\
\hline III & $7(22 \%)$ & & & \\
\hline \multicolumn{5}{|l|}{ CT-guided needle biopsy ${ }^{a}$} \\
\hline Yes & $8(33 \%)$ & 0.259 & 0.646 & 0.139 \\
\hline No & $16(67 \%)$ & & & \\
\hline $\mathrm{SUV}^{\mathrm{b}}$ range & $2.6-20.5$ & 0.691 & 0.863 & 0.537 \\
\hline
\end{tabular}

$\beta$-tubulin (cat. no. ZA-0581; dilution, 1:100), multidrug resistance-associated protein (cat. no. ZM-0345; dilution, 1:50) and P170 (cat. no. ZM-0189; dilution, 1:100; all from OriGene Technologies, Inc., Beijing, China). Immunohistochemical staining was performed on the $4-\mu \mathrm{m}$ formalin-fixed (at room temperature for $24 \mathrm{~h}$ ) paraffin-embedded tissue slides. Briefly, following deparaffinization and rehydration, using xylene and descending alcohol series, antigen retrieval was performed between 95 and $100^{\circ} \mathrm{C}$ citrate buffer $(10 \mu \mathrm{mol} / \mathrm{l}$, $\mathrm{pH}$ 6.0) for $15 \mathrm{~min}$. Subsequently, $3 \% \mathrm{H}_{2} \mathrm{O}_{2}$ in methanol was used to block endogenous peroxidase activity for $20 \mathrm{~min}$. Following blocking with goat serum (cat. no. ZLI-9022; OriGene Technologies, Inc.) in room temperature for $30 \mathrm{~min}$, the primary antibodies were added to the slides overnight at $4^{\circ} \mathrm{C}$. Subsequently, tissue sections were incubated with a secondary goat anti-mouse horseradish peroxidase (HRP)-conjugated antibody (cat. no. SAB3701044; dilution, 1:1,000; Sigma-Aldrich; Merck KGaA, Darmstadt, Germany) in room temperature for $30 \mathrm{~min}$. HRP activity was visualized using the Liquid DAB Plus Substrate kit (cat. no. ZLI-9018; OriGene Technologies, Inc.) according to manufacturer's protocol. Automated hematoxylin was used to counterstain the nucleus for $3 \mathrm{~min}$ at room temperature. Sections were examined under light microscope (magnification, x20).

Statistical analysis. Statistical analysis was performed using SPSS 17.0 for Windows (SPSS, Inc., Chicago, IL, USA). The association between CTC/CTM counts and clinical variables was analyzed with the Mann-Whitney (two groups) or Kruskal-Wallis ( $\geq 3$ groups) test for discrete variables, and 

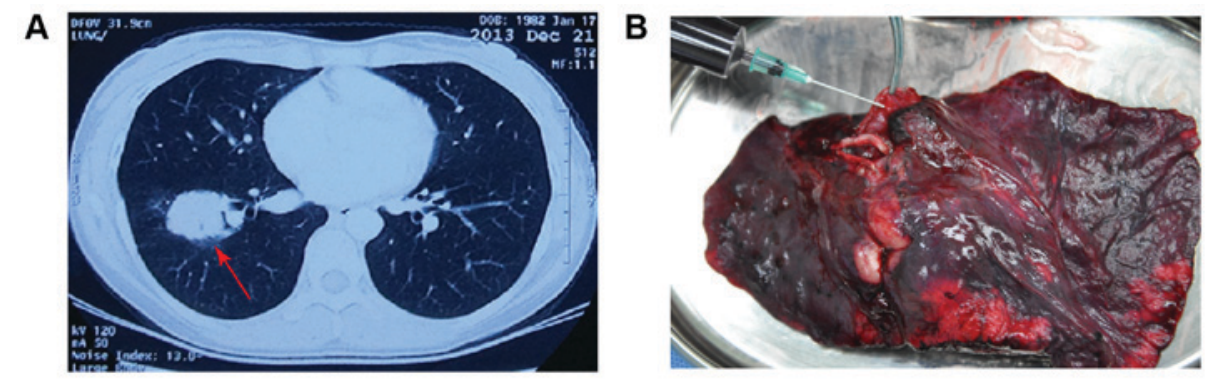

C

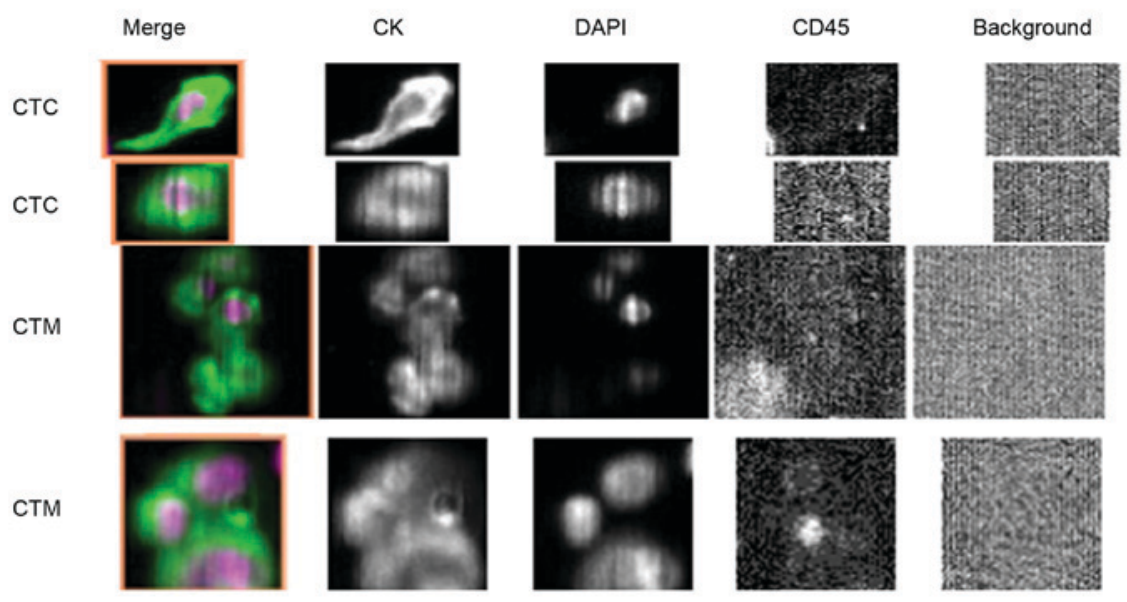

Figure 1. Blood collection and identification of CTCs and CTM. (A) Patient with a tumor in the right lower lobe of the lung (red arrow). (B) Upon lobectomy, a syringe was inserted into the pulmonary vein, and a $7.5-\mathrm{ml}$ blood sample was immediately collected. (C) Tumor cells were defined as those merged fluorescent images (from two patients) with round to oval morphology containing DAPI-stained nuclei and expressing CKs but not CD45. CTM were identified as CTC clusters containing $\geq 3$ nuclei (magnification, $\mathrm{x} 100$ ). CK, cytokeratin; CTC, circulating tumor cell; CTM, circulating tumor microemboli; CD45, cluster of differentiation 45 .

A

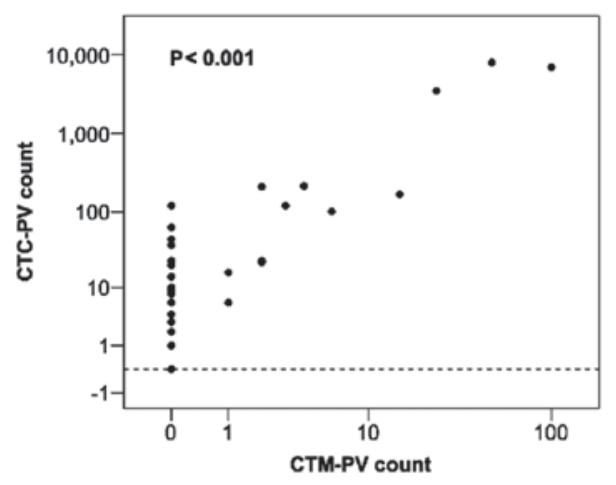

C

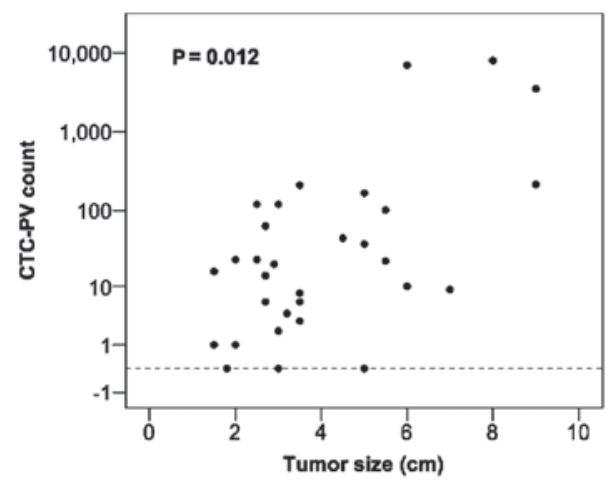

B

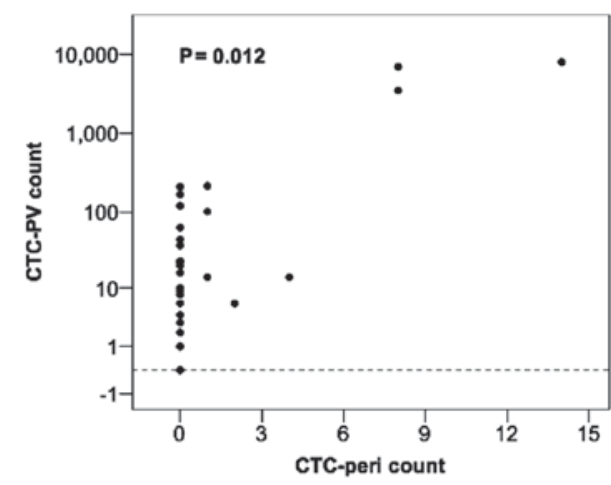

D

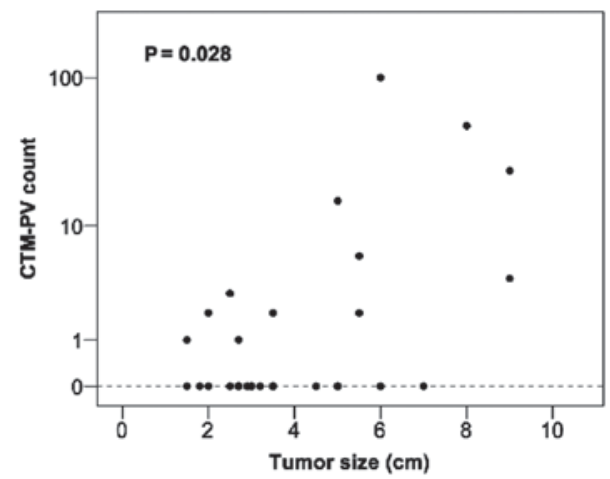

Figure 2. Correlation between CTC/CTM counts and clinical variables. Correlation analysis of CTC-PV with (A) CTM-PV and (B) CTC-peri counts. Association between (C) CTC-PV and (D) CTM-PV counts and tumor size. CTC, circulating tumor cell; CTM, circulating tumor microemboli; PV, pulmonary vein; peri, peripheral blood. 
A

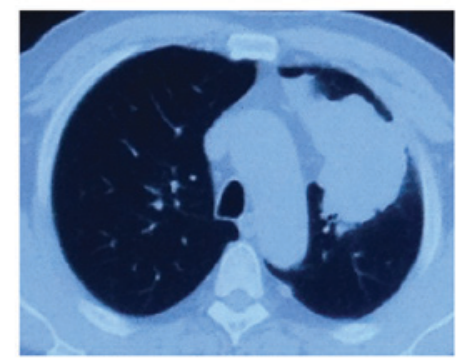

B

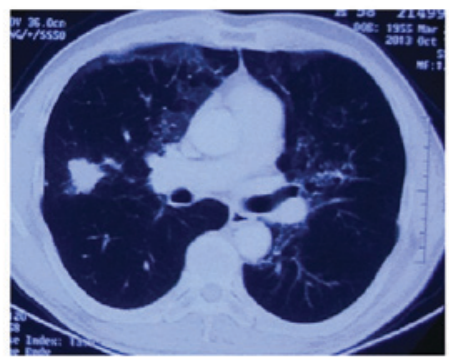

C

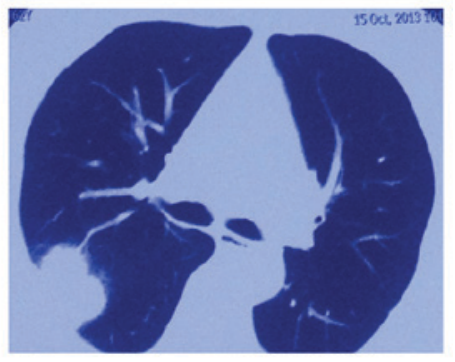

D

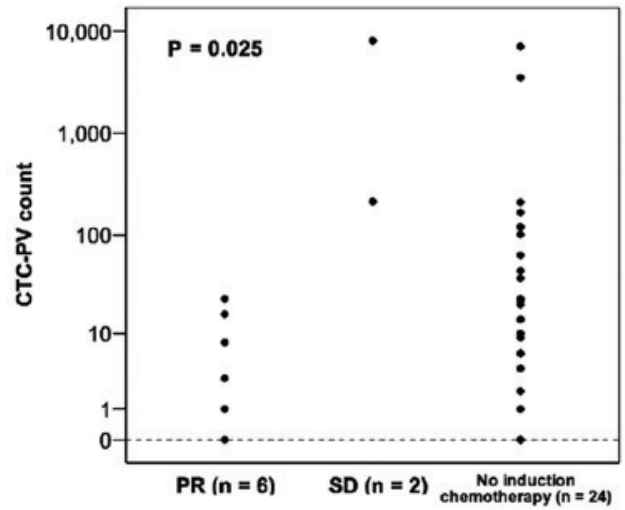

E

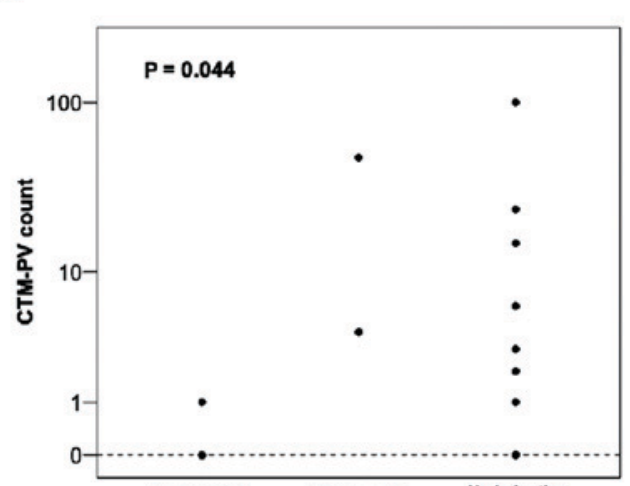

$\operatorname{PR}(n=6) \quad \operatorname{SD}(n=2) \quad \begin{gathered}\text { No induction } \\ \text { chemotherapy }(n=24)\end{gathered}$

H

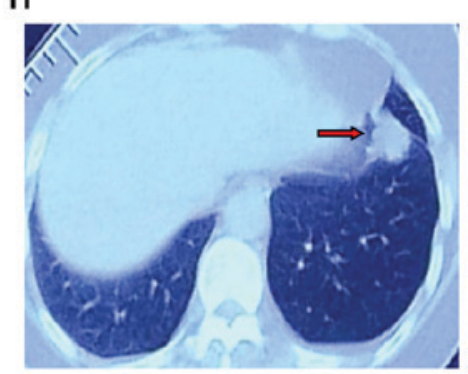

Figure 3. Patient computed tomography images and correlation between CTC/CTM counts and clinical variables. (A) The large tumor in the left upper lobe of the lung was $9.0 \mathrm{~cm}$ at its largest diameter and 3,500 CTCs and $24 \mathrm{CTM}$ were detected/7.5 ml PV blood following lobectomy in this patient. (B) A patient with lung cancer and a $2.5 \mathrm{~cm}$ tumor in the right middle lobe of the lung was pathologically diagnosed with stage IA disease. However, the CTC-PV count was 120 and the CTM-PV count was 3. (C) The PV blood from a patient with stage IB disease and a $5.0 \mathrm{~cm}$ tumor contained no CTCs or CTM. Distribution of (D) CTC-PV and (E) CTM-PV according to response to neoadjuvant chemotherapy. (F) Tumor in the left upper lobe of another patient with lung cancer (red arrow). (G) The tumor achieved PR following 2 cycles of induction chemotherapy with gemcitabine plus cisplatin (red arrow). Although the pathological stage was IIIA, there were only $8 \mathrm{CTC}-\mathrm{PV}$ and no CTM in this patient. (H) Another patient with stage IA disease $(2.0 \mathrm{~cm}$ tumor) without induction therapy had $23 \mathrm{CTC}-\mathrm{PV}$ and $2 \mathrm{CTM}-\mathrm{PV}$ (red arrow). CTC, circulating tumor cell; CTM, circulating tumor microemboli; PV, pulmonary vein; PR, partial response; SD, stable disease.

with Spearman's correlation analysis for continuous variables. $\mathrm{P}<0.05$ was considered to indicate a statistically significant difference.

\section{Results}

Detection of CTC-PV/CTM-PV and CTC-peri/CTM-peri counts. The majority the 32 patients with lung cancer had CTCs in their PV blood $(n=29,90.6 \%$; range, 1-8,000; median, 18; mean, 617). CTM-PV were also detected in 12 patients $(37.5 \%$; range, 1-100; median, 4; mean, 17). Only 8 patients had CTCs in their peripheral blood (25\%; range $=1-14)$, whereas no CTM-peri were detected. Neither CTCs nor CTM were detected in the PV or peripheral blood of the patient with pulmonary hamartoma. Correlation analysis revealed a significant association between the CTC-PV counts and CTM-PV
$(\mathrm{P}<0.001)$ and CTC-pericounts $(\mathrm{P}=0.012)$ (Fig. 2A and $\mathrm{B}$, respectively).

Correlation between CTC-PV/CTM-PV counts and clinical variables. Table I lists the association between CTC-PV/CTM-PV counts and the corresponding clinical parameters. Statistical analysis indicated that tumor size, lymph node metastasis and pathological staging were significantly correlated with increased CTC-PV counts. Furthermore, tumor size was also positively associated with increased CTM counts (Fig. 2C and D). Although patients with vessel invasion tended to have higher CTC-PV counts, this association was not significant $(\mathrm{P}=0.059)$. In addition, pre-surgery CT-guided needle biopsy and PET-CT evaluation of primary tumor SUV had no impact on CTC count (Table I). All 3 patients with >1,000 CTC-PV had large tumors 
Table II. CTC-PV/CTM-PV counts of 12 patients with lung cancer and small tumors $(<3.0 \mathrm{~cm})$.

\begin{tabular}{lccccc}
\hline $\begin{array}{l}\text { Patient } \\
\text { no. }\end{array}$ & $\begin{array}{c}\text { Tumor size } \\
(\mathrm{cm})\end{array}$ & LN & Stage & CTC-PV $^{\mathrm{a}}$ & CTM-PV $^{\mathrm{C}}$ \\
\hline 1 & 1.8 & N0 & IA & 0 & 0 \\
2 & 1.5 & N0 & IA & 1 & 0 \\
3 & 2.0 & N0 & IA & 1 & 0 \\
4 & 2.7 & N0 & IA & 6 & 1 \\
$5^{\mathrm{c}}$ & 2.0 & N0 & IA & 23 & 2 \\
$6^{\mathrm{d}}$ & 2.5 & N0 & IA & 120 & 3 \\
7 & 1.5 & N0 & IB & 16 & 1 \\
8 & 2.5 & N0 & IB & 23 & 0 \\
9 & 2.7 & N1 & IIA & 14 & 0 \\
10 & 2.7 & N1 & IIA & 14 & 0 \\
11 & 2.7 & N1 & IIA & 63 & 0 \\
12 & 2.9 & N2 & IIIA & 20 & 0 \\
\hline
\end{tabular}

${ }^{\mathrm{a}}$ Mean $\pm \mathrm{SD}, 23.8 \pm 40.1 ; \mathrm{P}=0.495 .{ }^{\mathrm{b}}$ Mean $\pm \mathrm{SD}, 0.9 \pm 1.1 ; \mathrm{P}=0.105$. ${ }^{\mathrm{C}}$ The CT image of this patient is shown in Fig. 3H. ${ }^{\mathrm{d}}$ The CT image of this patient is shown in Fig. 3B. LN, lymph node; CTC, circulating tumor cell; CTM, circulating tumor microemboli; PV, pulmonary vein; SD, standard deviation; CT, computed tomography.

$(>6.0 \mathrm{~cm})$. Fig. 3A demonstrates the CT images of a patient with a large primary tumor. However, patients with small tumors also had considerable CTC counts in their PV blood regardless of their pathological stage $(\mathrm{P}=0.495$; Table II). Table II lists the CTC-PV/CTM-PV counts and clinical characteristics of 12 patients with tumors $<3.0 \mathrm{~cm}$. The median and mean CTC-PV counts of these 12 patients were 15 and 25 , respectively. Fig. $3 \mathrm{~B}$ and $\mathrm{C}$ demonstrate the $\mathrm{CT}$ images of patients with varying tumor sizes.

Perioperative treatment, CTC/CTM counts and survival result. Of the 32 patients, 8 received platinum-based chemotherapy prior to surgery, and partial response (PR) was noted in 6 patients. Table III lists the CTC/CTM counts in 8 patients who received neoadjuvant chemotherapy. Statistical analysis revealed that the CTC-PV and CTM-PV counts in the PR cases were significantly lower than those in patients with stable disease (SD) or patients who did not receive induction therapy. $(\mathrm{P}=0.025$ and $\mathrm{P}=0.044$, respectively; Fig. 3D and E). Fig. 3F-H demonstrate the CT images of patients who had or had not undergone induction chemotherapy. Upon surgery, 11 patients received four cycles of platinum-based adjuvant chemotherapy based on their pathological stage, while another 5 patients diagnosed with stage II-IIIA adenocarcinoma with EGFR mutation were enrolled in an ongoing phase-II study and received adjuvant targeted therapy (icotinib). The median follow-up time was 12 months; 4 patients recurred postoperatively, 3 of whom succumbed to the disease. All 4 patients had received perioperative therapy, and their CTC-PV and CTM-PV counts are listed in Table IV. Compared with the patients who did not experience a recurrence, no significant correlation was observed between CTC-PV/CTM-PV counts and disease recurrence $(\mathrm{P}=0.266$ and $\mathrm{P}=0.394$, respectively; Table IV).
Xenograft assay and tumor analysis. PV blood $(15 \mathrm{ml})$ was collected from 3 patients in the overall cohort to perform a xenograft assay. Table $\mathrm{V}$ lists the patient characteristics and CTC/CTM counts. The PV blood of one of the patients, who had 167 CTCs and 15 CTM per $7.5 \mathrm{ml}$ PV blood as detected by CELLSEARCH, formed a subcutaneous xenograft tumor in the injected mouse. Fig. 4A depicts CT images of a patient and a mouse xenograft tumor. H\&E staining, and CK7, CK20 and TTF-1 IHC results revealed concordant typical adenocarcinoma between the primary tumor and the matched xenograft. In addition, there was similar positive expression of drug resistance-associated proteins in the xenograft tumor compared with that in the patient. Fig. $4 \mathrm{~B}$ and $\mathrm{C}$ demonstrate a series of H\&E and IHC results of a patient primary tumor and the xenograft tumor.

\section{Discussion}

The present study detected numerous CTCs and CTM in $7.5 \mathrm{ml}$ PV blood compared with those in peripheral blood. Although previous studies have demonstrated that intraoperative manipulation increases hematogenous dissemination in patients with NSCLC $(5,6,17)$, whether the PV should be ligated first remains controversial. Hashimoto et al reported that the increased CTC-PV count prior and subsequent to surgical manipulation for lobectomy was not significantly associated with the sequence of vessel interruption (12). Refaely et al also demonstrated that the sequence of vessel interruption was not a risk factor for recurrence (18). Therefore, ligating the pulmonary artery first remains an option during lobectomy based on the preference of the surgeon (11) or the minimally invasive surgery technique used, particularly for upper lobectomy (19). Different from a previous study (11), the PV was stapled prior to other surgical manipulation in the present study. Following lobectomy, a large number of CTCs and CTM were retained and detected in PV blood. Okumura et al and Hashimoto et al reported no significant correlation between CTC-PV count and patient characteristics $(11,12)$. However, the present study observed an apparent increase in CTC-PV in patients with large primary tumors and lymph node metastasis, which indicated that, for these patients, surgical behavior such as retracting the lobe, exposing the hilum or manipulating the tumor may present a high risk for disseminating tumor cells into the blood during surgery. The present study also analyzed the CTC-PV count in 12 patients with small tumors $(<3.0 \mathrm{~cm})$, and noticed that the majority of patients had CTCs in their PV blood ( $\mathrm{n}=11,91.7 \%)$. Furthermore, 8 of these 12 patients were diagnosed as pathological stage I (stage IA, 6; stage IB, 2), but 50\% of them were CTM-positive $(n=4,50.0 \%)$. Although the immune system would clear the majority of tumor cells shed into the blood, and only a small portion of CTCs can develop metastases (20), several studies have demonstrated that CTCs detected in PV blood predict poor clinical outcome $(21,22)$. Therefore, even though the impact of intraoperative manipulation on survival remains unclear, lobectomy may be recommended for lung cancer of any tumor size and stage according to oncological principles, in addition to ligating the $\mathrm{PV}$, if possible, prior to any other treatment.

Besides surgical resection, the correlation between CTC-PV counts and the outcome of perioperative treatment, 
Table III. CTC-PV/CTM-PV counts of 8 patients with lung cancer who received induction chemotherapy.

\begin{tabular}{|c|c|c|c|c|c|c|c|c|c|}
\hline $\begin{array}{l}\text { Patient } \\
\text { no. }\end{array}$ & Histology & cTNM & $\begin{array}{c}\text { Chemotherapy } \\
\text { regimen }\end{array}$ & $\begin{array}{l}\text { No. of } \\
\text { cycles }\end{array}$ & Response & ypTNM & $\begin{array}{l}\text { Residual } \\
\text { tumor }(\%)\end{array}$ & CTC-PV & CTM-PV \\
\hline 1 & Squamous & cT2bN0M0 & GP & 1 & PR & ypT1bN0M0 & 20 & 0 & 0 \\
\hline 2 & Adenocarcinoma & cT2aN0M0 & $\mathrm{TP}$ & 3 & PR & ypT1aN0M0 & 95 & 1 & 0 \\
\hline 3 & Squamous & cT3N1M0 & GP & 2 & PR & ypT3N0M0 & 95 & 3 & 0 \\
\hline $4^{\mathrm{a}}$ & Squamous & cT2aN2M0 & GP & 2 & PR & ypT2aN2M0 & 95 & 8 & 0 \\
\hline 5 & Squamous & cT3N0M0 & GP & 2 & PR & ypT2aN0M0 & 95 & 16 & 1 \\
\hline 6 & Squamous & cT3N2M0 & GP & 2 & PR & ypT2aN0M0 & 80 & 23 & 0 \\
\hline 7 & Large cell & cT3N1M0 & GP & 2 & SD & ypT3N0M0 & 30 & 214 & 4 \\
\hline 8 & Squamous & cT3N1M0 & GP & 2 & SD & ypT3N1M0 & 80 & 8,000 & 48 \\
\hline
\end{tabular}

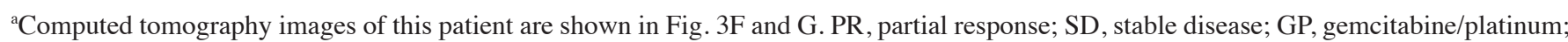
TP, paclitaxel/platinum; cTNM, clinical tumor-node-metastasis; ypTNM, pathological tumor-node-metastasis following therapy; CTC, circulating tumor cell; CTM, circulating tumor microemboli; PV, pulmonary vein.

Table IV. CTC-PV/CTM-PV counts of 4 patients with lung cancer andpostoperative recurrence.

\begin{tabular}{llclrrrr}
\hline Patient no. & Histology & Tumor size $(\mathrm{cm})$ & Stage & CTC-PV & CTM-PV & DFS (months) & OS (months) \\
\hline 1 & Squamous & 7.0 & pT2bN0M0 & 9 & 0 & 7 & 10 \\
2 & Squamous & 2.5 & ypT2aN0M0 & 23 & 0 & 3 & 4 \\
3 & Adenocarcinoma & 5.5 & pT2bN2M0 & 101 & 6 & 2 & 6 \\
$4^{\text {a }}$ & Adenocarcinoma & 9.0 & pT3N0M0 & 3,500 & 24 & 13 & Alive \\
\hline
\end{tabular}

${ }^{a}$ The computed tomographyimage of this patient is shown in Fig.3A. CTC, circulating tumor cell; CTM, circulating tumor microemboli; PV, pulmonary vein; DFS, disease-free survival; OS, overall survival.

Table V. CTC-PV/CTM-PV counts of 3 cases of lung cancer whose PV blood was used for xenograft assay.

\begin{tabular}{lcccrrr}
\hline Patient no. & Histology & Tumor size $(\mathrm{cm})$ & Stage & CTC-PV & CTM-PV & Successful xenograft tumor \\
\hline 1 & Adenocarcinoma & 5.5 & pT2bN2M0 & 101 & 6 & No \\
2 & Adenocarcinoma & 5.0 & pT2aN1M0 & 167 & 15 & Yes \\
3 & Squamous & 3.5 & ypT3N0M0 & 3 & 0 & No \\
\hline
\end{tabular}

CTC, circulating tumor cell; CTM, circulating tumor microemboli; PV, pulmonary vein.

particularly induction chemotherapy, was also analyzed in the present study. Despite the fact that only 8 patients received platinum-based chemotherapy prior to surgery, a promising tendency for the CTC count to markedly decrease was noted in patients who achieved response through induction therapy compared with that in other patients. The pathological result revealed that the majority of patients had $>80 \%$ of residual tumor, but the patient whose tumor size was $3.0 \mathrm{~cm}$ with only $20 \%$ of residual tumor had no CTCs or CTM in his PV blood. Previous studies have reported that the percentage of viable tumor cells is a significant predictor of overall survival and disease-free survival in patients with neoadjuvant-treated NSCLC, but not in patients who undergo surgery alone $(23,24)$. It can be hypothesized that the neoadjuvant chemotherapy-mediated inhibition of tumor cell metastatic characteristics and changes in the percentage of tumor cells in primary disease reduces CTC shedding into the blood during surgery. In addition, it has been reported that tumor cells within CTM have survival advantage and relative resistance to cytotoxic drugs (16). Consistently, the present study revealed that, among 6 PR cases, 5 were free from CTM-PV. The CTM-PV count in PR cases was significantly less than that in SD cases or patients who did not receive induction therapy. This finding implies that CTCs/CTM in PV blood may indirectly determine the response to induction therapy, and suggests to a certain degree, that neoadjuvant treatment contributes to the reduction of intraoperative hematogenous dissemination in patients with locally advanced disease or heavy tumor burden. As the follow-up period was short, the correlation between CTC-PV count and disease recurrence or the postoperative result of 
A
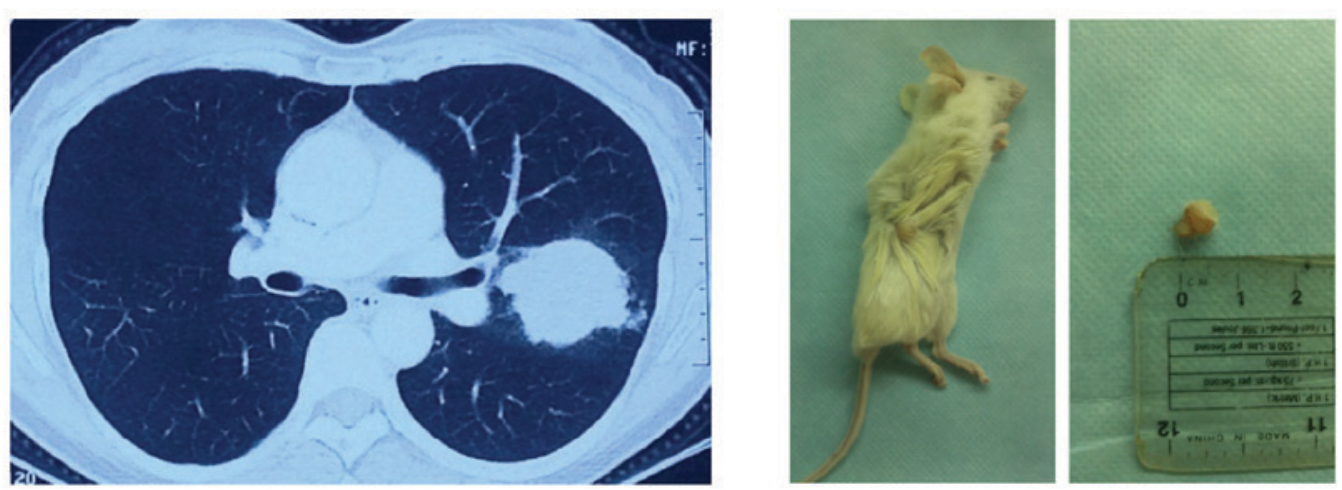

B

H\&E

CK7

CK20

TTF-1
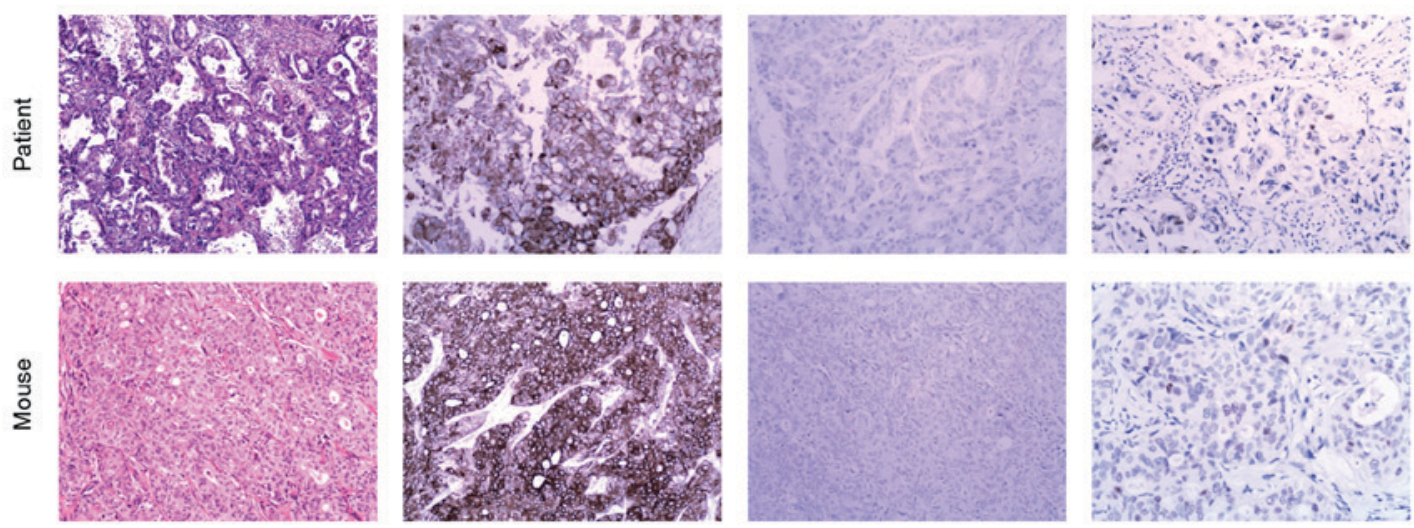

C
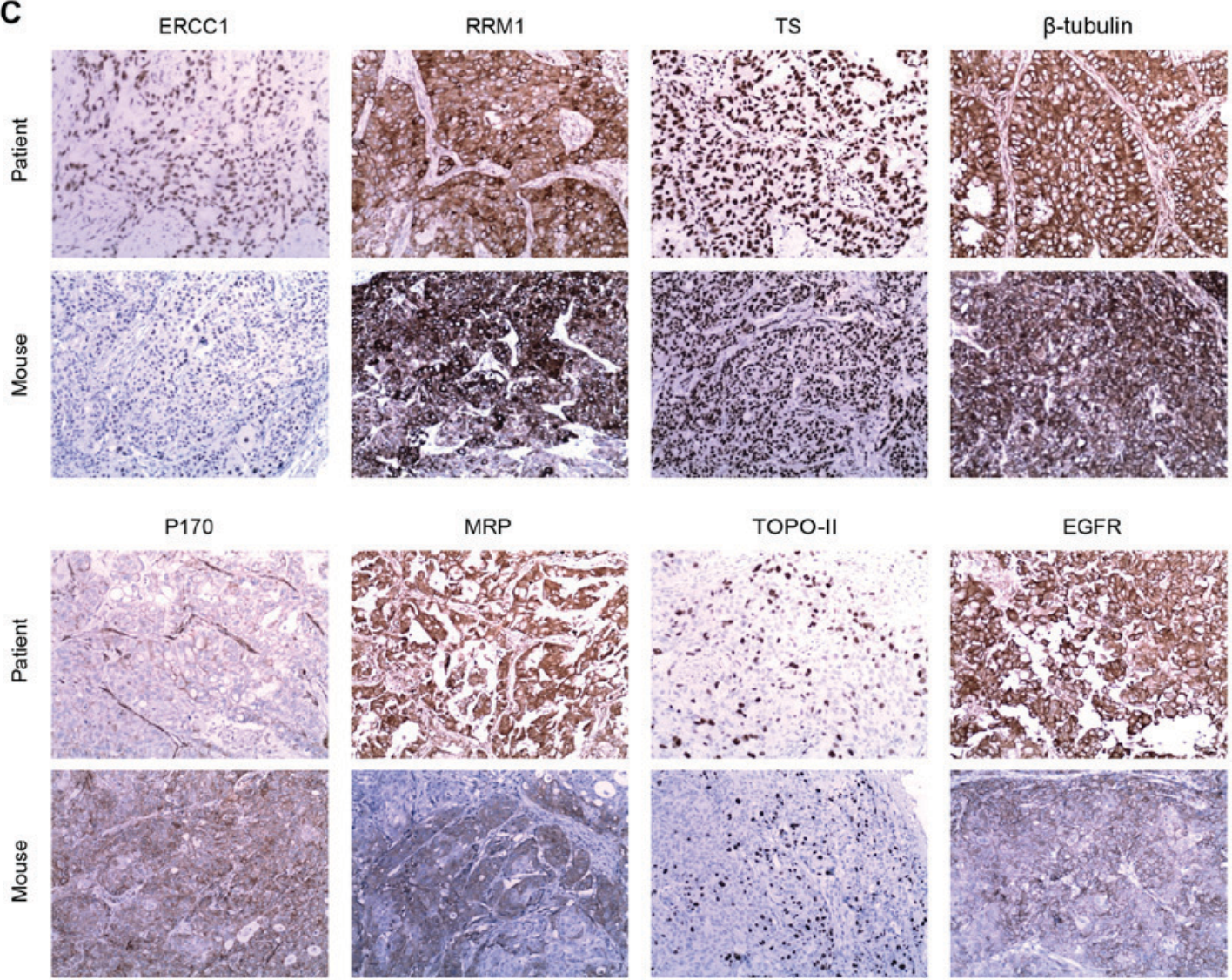

Figure 4. Xenograft assay and IHC staining. (A) Patient with a tumor in the left upper lobe of the lung (left panel) and mouse xenograft tumor formed following injection with circulating tumor cells from the pulmonary vein of the patient (right panel). (B) H\&E and CK7-positive, CK20-negative and TTF-1-positive IHC staining of the primary tumor from the patient and the mouse xenograft tumor. (C) IHC staining of a series of drug resistance-associated proteins in the primary and xenograft tumors (magnification, x20). H\&E, hematoxylin and eosin; CK, cytokeratin; TTF-1, thyroid nuclear factor-1; ERCC1, excision repair cross-complementing 1; RRM1, ribonucleotide reductase M1; TS, thymidylate synthase; MRP, multi-drug resistance-associated protein; TOPO-II, topoisomerase II; EGFR, epidermal growth factor receptor; IHC, immunohistochemistry. 
adjuvant therapy was not determined. It should be mentioned that CTC/CTM counts are not always consistent with clinical characteristics, which indicates that tumor cell shedding into the blood is not only influenced by TNM stage, but is also associated with tumor biological behavior. Therefore, in addition to TNM staging, future studies should determine whether CTC and CTM counts could guide adjuvant chemotherapy following surgery.

Although the CELLSEARCH system has been approved in the USA by the FDA for monitoring hematogenous metastasis in patients with cancer, no direct evidence indicates that the captured CTCs are true tumor cells and that they are able to form distant metastases (11). In the present study, the results of a xenograft assay demonstrated that the CTCs and CTM detected in the PV blood of patients were tumorigenic. Similar human CTC-derived xenograft models have been reported for breast cancer, hepatocellular carcinoma and SCLC (25-27). In addition, CTC culture has been established successfully from patients with early-stage NSCLC (28). An additional assay was performed in the present study to compare differences in protein expression between the primary tumor and the xenograft model in order to explore whether the mouse xenograft tumor derived from CTC-PV differed from that of the patient in terms of drug resistance. However, there were various limitations, as the xenograft assay was performed using cells from only 3 patients, and only 1mouse developed a xenograft tumor. To confirm these results, future studies should enroll additional patients for xenograft assay and cell culture to explore CTC-PV tumorigenicity and to analyze its tumor biological features.

To conclude, numerous CTC-PV/CTM-PV are tumorigenic and correlate positively with tumor size and stage. Despite the limited case number and short follow-up time, the present study provides evidence for adhering to oncological principles in lung cancer surgery, and reports a potential biomarker for guiding perioperative treatment. The impact of surgical behavior on survival will be evaluated in further studies.

\section{Acknowledgements}

The present study was supported by grants from Peking University (PKU) 985 Special Funding for Collaborative Research with PKU Hospitals, the National High Technology Research and Development Program of China (863 Program; grant no. 2012AA02A502) and Beijing Municipal Administration of Hospital Clinical Medicine Development of Special Funding (grant no. ZYLX201509).

\section{References}

1. Siegel R, Naishadham D and Jemal A: Cancer statistics, 2013. CA-Cancer J Clin 63: 11-30, 2013.

2. Ramalingam SS, Owonikoko TK and Khuri FR: Lung cancer: New biological insights and recent therapeutic advances. CA Cancer J Clin 61: 91-112, 2011.

3. Goldstraw P, Crowley J, Chansky K, Giroux DJ, Groome PA, Rami-Porta R, Postmus PE, Rusch V and Sobin L; International Association for the Study of Lung Cancer International Staging Committee; Participating Institutions: The IASLC lung cancer staging project: Proposals for the revision of the TNM stage groupings in the forthcoming (seventh) edition of the TNM Classification of malignant tumours. J Thorac Oncol 2: 706-714, 2007.
4. Pantel K, Brakenhoff RH and Brandt B: Detection, clinical relevance and specific biological properties of disseminating tumour cells. Nat Rev Cancer 8: 329-340, 2008.

5. Yamashita JI, Kurusu Y, Fujino N, Saisyoji T and Ogawa M: Detection of circulating tumor cells in patients with non-small cell lung cancer undergoing lobectomy by video-assisted thoracic surgery: A potential hazard for intraoperative hematogenous tumor cell dissemination. J Thorac Cardiovasc Surg 119: 899-905, 2000.

6. Dong Q, Huang J, Zhou Y, Li L, Bao G, Feng J and Sha H: Hematogenous dissemination of lung cancer cells during surgery: Quantitative detection by flow cytometry and prognostic significance. Lung Cancer 37: 293-301, 2002.

7. Allard WJ, Matera J, Miller MC, Repollet M, Connelly MC, Rao C, Tibbe AG, Uhr JW and Terstappen LW: Tumor cells circulate in the peripheral blood of all major carcinomas but not in healthy subjects or patients with nonmalignant diseases. Clin Cancer Res 10: 6897-6904, 2004.

8. Cristofanilli M, Budd GT, Ellis MJ, Stopeck A, Matera J, Miller MC, Reuben JM, Doyle GV, Allard WJ, Terstappen LW and Hayes DF: Circulating tumor cells, disease progression, and survival in metastatic breast cancer. N Engl J Med 351: 781-791, 2004.

9. Tanaka F, Yoneda K, Kondo N, Hashimoto M, Takuwa T, Matsumoto S, Okumura Y, Rahman S, Tsubota N, Tsujimura T, et al: Circulating tumor cell as a diagnostic marker in primary lung cancer. Clin Cancer Res 15: 6980-6986, 2009.

10. Krebs MG, Sloane R, Priest L, Lancashire L, Hou JM, Greystoke A, Ward TH, Ferraldeschi R, Hughes A, Clack G, et al: Evaluation and prognostic significance of circulating tumor cells in patients with non-small-cell lung cancer. J Clin Oncol 29: 1556-1563, 2011.

11. Okumura Y, Tanaka F, Yoneda K, Hashimoto M, Takuwa T, Kondo $\mathrm{N}$ and Hasegawa $\mathrm{S}$ : Circulating tumor cells in pulmonary venous blood of primary lung cancer patients. Ann Thorac Surg 87: 1669-1675, 2009.

12. Hashimoto M, Tanaka F, Yoneda K, Takuwa T, Matsumoto S, Okumura Y, Kondo N, Tsubota N, Tsujimura T, Tabata C, et al: Significant increase in circulating tumour cells in pulmonary venous blood during surgical manipulation in patients with primary lung cancer. Interact Cardiovasc Thorac Surg 18: 775-783, 2014.

13. Rami-Porta R, Wittekind $\mathrm{C}$ and Goldstraw P; International Association for the Study of Lung Cancer (IASLC) Staging Committee: Complete resection in lung cancer surgery: Proposed definition. Lung Cancer 49: 25-33, 2005.

14. Eisenhauer EA, Therasse P, Bogaerts J, Schwartz LH, Sargent D, Ford R, Dancey J, Arbuck S, Gwyther S, Mooney M, et al: New response evaluation criteria in solid tumours: Revised RECIST guideline (version 1.1). Eur J Cancer 45: 228-247, 2009.

15. Mountain CF and Dresler CM: Regional lymph node classification for lung cancer staging. Chest 111: 1718-1723, 1997.

16. Hou JM, Krebs MG, Lancashire L, Sloane R, Backen A, Swain RK, Priest LJ, Greystoke A,Zhou C, Morris K, et al: Clinical significance and molecular characteristics of circulating tumor cells and circulating tumor microemboli in patients with small-cell lung cancer. J Clin Oncol 30: 525-532, 2012.

17. Kurusu Y, Yamashita J, Hayashi N, Mita S, Fujino N and Ogawa M: The sequence of vessel ligation affects tumor release into the circulation. J Thorac Cardiovasc Surg 116: 107-113, 1998.

18. Refaely Y, Sadetzki S, Chetrit A, Simansky DA, Paley M, Modan B and Yellin A: The sequence of vessel interruption during lobectomy for non-small cell lung cancer: Is it indeed important? J Thorac Cardiovasc Surg 125: 1313-1320, 2003.

19. Fieira Costa E, Delgado Roel M, Paradela de la Morena M, Gonzalez-Rivas D, Fernandez-Prado R and de la Torre M: Technique of uniportal VATS major pulmonary resections. J Thorac Dis 6 (Suppl 6): S660-S664, 2014.

20. Glaves D: Correlarion between circulating cancer cells and incidence of metastases. Br J Cancer 48: 665-673, 1983.

21. Sienel W, Seen-Hibler R, Mutschler W, Pantel K and Passlick B: Tumour cells in the tumour draining vein of patients with non-small cell lung cancer: Detection rate and clinical significance. Eur J Cardiothorac Surg 23: 451-456, 2003.

22. Funaki S, Sawabata N, Nakagiri T, Shintani Y, Inoue M, Kadota Y, Minami M and Okumura M: Novel approachfor detection of isolated tumor cells in pulmonary vein using negative selection method: Morphological classification and clinical implications. Eur J Cardiothorac Surg 40: 322-327, 2011. 
23. Pataer A, Kalhor N, Correa AM, Raso MG, Erasmus JJ, Kim ES, Behrens C, Lee JJ, Roth JA, Stewart DJ, et al: Histopathologic response criteria predict survival of patients with resected lung cancer after neoadjuvant chemotherapy. J Thorac Oncol 7: $825-832,2012$.

24. William WN Jr, Pataer A, Kalhor N, Correa AM, Rice DC, Wistuba II,Heymach J,Lee JJ,Kim ES, Munden R, et al: Computed tomography RECIST assessment of histopathologic response and prediction of survival in patients with resectable non-small-cell lung cancer after neoadjuvant chemotherapy. J Thorac Oncol 8: 222-228, 2013.

25. Baccelli I, Schneeweiss A, Riethdorf S, Stenzinger A, Schillert A, Vogel V, Klein C, Saini M, Bäuerle T, Wallwiener M, et al: Identification of a population of blood circulating tumor cells from breastcancerpatientsthatinitiatesmetastasisinaxenograftassay.Nat Biotechnol 31: 539-544, 2013.
26. Sun YF, Xu Y, Yang XR, Guo W, Zhang X, Qiu SJ, Shi RY, Hu B, Zhou J and Fan J: Circulating stem cell-like epithelial cell adhesion molecule-positive tumor cells indicate poor prognosis of hepatocellular carcinoma after curative resection. Hepatology 57: 1458-1468, 2013

27. Hodgkinson CL, Morrow CJ, Li Y, Metcalf RL, Rothwell DG, Trapani F, Polanski R, Burt DJ, Simpson KL, Morris K, et al: Tumorigenicity and genetic profiling of circulating tumor cells in small-cell lung cancer. Nat Med 20: 897-903, 2014.

28. Zhang Z, Shiratsuchi H, Lin J, Chen G, Reddy RM, Azizi E, Fouladdel S, Chang AC, Lin L, Jiang $\mathrm{H}$, et al: Expansion of CTCs from early stage lung cancer patients using a microfluidic co-culture model. Oncotarget 5: 12383-12397, 2014. 